\title{
SÍNTOMAS NEGATIVOS DE LA ESQUIZOFRENIA: AUTOPSIA PSICOLÓGICA DE CHRISTOPHER JOHNSON MCCANDLESS
}

NEGATIVE SYMPTOMS OF SCHIZOPHRENIA: PSYCHOLOGICAL AUTOPSY OF CHRISTOPHER JOHNSON MCCANDLESS

ERNESTO FLORES SIERRA*

Recibido: 29 de marzo de 2016 Aprobado: 6 de junio de 2016 



\section{SÍNTOMAS NEGATIVOS DE LA ESQUIZOFRENIA: AUTOPSIA PSICOLÓGICA DE CHRISTOPHER JOHNSON MCCANDLESS}

\section{Ernesto Flores Sierra}

PALABRAS CLAVES: Esquizofrenia, síntomas negativos, autopsia psicológica, psicopatología.

KEY WORDS: Schizophrenia, negative symptoms, psychological autopsy, psychopathology.

\section{RESUMEN}

El presente artículo consiste en el desarrollo de la autopsia psicológica de Christopher Johson McCandless, para lo cual hemos realizado una descripción de la sintomatología negativa de la esquizofrenia, considerando para dicha descripción tanto las propuestas psicopatológicas clásicas, como las nuevas propuestas clínicas. Con este insumo hemos analizado los rasgos conductuales de McCandless en base a los datos recogidos en su biografía "Hacia tierras salvajes". Como producto se propone la autopsia psicológica como una herramienta en el campo de la psicología patológica y forense y se realiza un estudio de la sintomatología negativa de los cuadros esquizofrénicos y esquizoides.

\section{ABSTRACT}

This article makes a psychological autopsy of Christopher McCandless, we made a description of the negative symptoms of schizophrenia, considering for this description classical psychopathological proposals, and new clinical proposals. With this input, we have analyzed the behavioral traits of McCandless based on data collected in his biography "Into the wild". As a result the psychological autopsy is a instrument in the field of pathological and forensic psychology and a study of negative symptoms of schizophrenia and schizoid pictures is performed. 


\section{INTRODUCCIÓN}

La sintomatología de la esquizofrenia ha sido ampliamente descrita por la investigación psicopatológica, y se ha determinado una serie de formas de conducta alterada específicas de este trastorno que se presentan dependiendo del tipo, curso o momento de inicio del cuadro. Sin embargo, los síntomas llamados negativos (Andreasen, 1982), suelen ser aquellos que se prolongan en el tiempo y están presentes en todas las variantes de la enfermedad, se presentan antes de los primeros brotes psicóticos como en el caso de autismo esquizofrénico o en la personalidad paranoide (Chinchilla Moreno, A., Quintero Gutiérrez del Álamo, J., Correas Lauffer, J. , 2011) y continúan presentándose cuando la enfermedad se encuentra en su fase crónica o bien en su fase residual (Sluchevsky, 1960/1984). Es importante mencionar que si bien los fármacos típicos como los inhibidores selectivos de la recapatación de serotonina, tienen un efecto importante en el control de los síntomas positivos de la enfermedad, su acción es reducida o nula sobre los síntomas negativos. (Chinchilla Moreno, A., Quintero Gutiérrez del Álamo, J., Correas Lauffer, J., 2011).

Christopher McCandless es famoso en la historia reciente de los Estados Unidos por haber sido encontrado muerto en medio de los bosques de Alaska tras 4 meses de vivir totalmente aislado de la sociedad, la causa de su fallecimiento fue inanición, y el lugar donde murió se ha convertido en un lugar de culto para trotamundos y aventureros que atraídos por su historia repiten su viaje hacia los bosques subárticos. Su historia se encuentra contenida en el libro Into the wild, del periodista Jon Krakauer, publicado en castellano como Hacia rutas salvajes, en el año 2008. Dicho relato se encuentra basado en los diarios de McCandless, en entrevistas a sus familiares y amigos, y en los testimonios de quienes lo conocieron antes que se interne voluntariamente en la naturaleza para vivir aislado de la sociedad. Si bien Krakauer en el texto va a afirmar que McCandles no padecía un trastorno mental, y que su muerte se produjo por una intoxicación con una semilla de tubérculo de la región, nuestra propuesta consiste en indagar la presencia de síntomas negativos de esquizofrenia en el famoso trotamundos, como una forma de explicar las características de su deceso.

La metodología que se utilizará será la de describir aspectos psicológicos y conductuales de McCandless y analizar si los mismos se corresponden con los síntomas negativos de la esquizofrenia 
ReVista PUCE. ISSN 1390-7719. Núm.103. 3 de mayo de 2016 -

3 De nOViembre De 2016. ERnesto Flores SierRa. PP. 33-63

descritos por la psicopatología actual, para establecer un perfil psicológico del sujeto investigado.

El presente trabajo de investigación buscará de esta manera cumplir dos propósitos, primero explorar la semiología negativa de la esquizofrenia, considerando que las características fundamentales de la enfermedad radican en la presencia de esta sintomatología crónica y resistente a la medicación; y luego realizar una autopsia psicológica de Alex Supertramp (seudónimo utilizado por McCandless) que nos permita aclarar las circunstancias de su famosa muerte.

\section{SÍNTOMAS NEGATIVOS DE LA ESQUIZOFRENIA.}

El DSM- 5 en correlación con ediciones anteriores del manual de diagnóstico va a considerar que existen cinco tipos de síntomas psicóticos por excelencia: ideas delirantes, lenguaje desorganizado, alucinaciones, comportamiento desorganizado y síntomas negativos. Para emitir un diagnóstico, el manual de la APA exige la presencia de al menos dos de estos síntomas que no se puedan explicar mejor por la presencia de otro trastorno mental, consumo de sustancias o enfermedad orgánica.

Los síntomas negativos son definidos como:

Los síntomas negativos incluyen una variedad limitada de expresiones de emoción (afecto aplanado o limitado), reducción notable de la cantidad o la fluidez del lenguaje y pérdida del deseo de hacer cosas (abulia). Se denomi- nan negativos debido a que dan la impresión de que algo ha sido sustraído del paciente, no agregado como sería el caso de las alucinaciones e ideas delirantes. Los síntomas negativos reducen la riqueza de texturas evidente de la personalidad de un paciente. Sin embargo, pueden ser difíciles de diferenciar de la actitud insulsa de la depresión, del consumo de drogas o de la falta de interés ordinaria. (Morrison, 2015)

Estos síntomas se encuentran relacionados con lo que se ha definido en la psicopatología actual como trastorno de personalidad esquizoide, el mismo que se caracteriza por la ausencia de interés en las relaciones sociales, rango emocional restringido, indiferencia, y una marcada tendencia a la soledad, y a evitar relaciones sociales estrechas. 
(Morrison, 2015). Para poder emitir un diagnóstico, se exige que estos síntomas estén presentes desde el inicio de la vida adulta y deben afectar gravemente la vida del sujeto en los ámbitos familiar, social y laboral.

Las personas con trastorno de personalidad esquizoide (TPE) se muestran indiferentes a la asociación con otras personas, en ocasiones en gran medida. De manera característica son solitarios de toda la vida, que cuentan con un rango emocional restringido, parecen insociables, fríos y huraños. Los individuos con TPE pueden tener éxito en empleos solitarios que otros encuentran difíciles de tolerar. Pueden soñar despiertos en exceso, tener gran apego a los animales y con frecuencia no se casan o no tienen relaciones románticas duraderas. Conservan el contacto con la realidad, a menos que desarrollen esquizofrenia. Sin embargo, sus parientes no tienen un riesgo más alto de padecer el trastorno. (Morrison, 2015)

Para poder establecer un diagnóstico es necesario que los síntomas mencionados se encuentren presentes, por lo menos durante seis meses de manera continua y debe existir la presencia premórbida de alteraciones en el desempeño social y laboral, y la enfermedad debe presentarse entre los 25 y los 40 años. (Morrison, 2015). Es importante notar que existe una comorbilidad im- portante entre el trastorno esquizoide de personalidad y la esquizofrenia, siendo el primero la base de personalidad sobre el que se desarrolla el segundo, y pasando a ser muchas veces una etapa previa al desarrollo de la esquizofrenia. Se menciona que antes de desarrollar la enfermedad el sujeto debe presentar una personalidad retraída y particular (esquizoide) y que debe existir generalmente un período prodrómico donde se comienzan a manifestar los síntomas psicóticos, el mismo que puede durar entre 3 y 6 años. La patología tiene un inicio gradual, que generalmente es imperceptible, caracterizada por la presencia de semiología negativa e ideas peculiares que no llegan a ser propiamente psicóticas. Los varones tienden a desarrollar de manera temprana la esquizofrenia; los síntomas se presentan toda la vida (en la mayoría de los casos), y la incapacidad para el desempeño social, familiar y laboral; suele instalarse de manera profunda desde el pródromo hasta el fin de la vida de la persona. (APA, 2014) Además de los cinco grandes síntomas psicóticos, las personas esquizofrénicas pueden presentar disfunciones cognitivas, como fuertes tendencias a la distracción y desorientación, estados de ánimo alterados con reacciones afectivas intensas e inapropiadas, ausencia de conciencia de la enfermedad, trastornos del sueño, y se menciona un índice epidemiológico de 
ReVista PUCE. ISSN 1390-7719. Núm.103. 3 de mayo de 2016 -

3 De nOViembre De 2016. ERnesto Flores SierRa. PP. 33-63

un $10 \%$ de pacientes esquizofrénicos varones que se suicidan (Morrison, 2015).

En lo que se refiere a los delirios, si bien existen una serie de formas de manifestación, muchas de las cuales se caracterizan por ser floridos e incoherentes, sus características fundamentales consisten en que los mismos son adoptados por el sujeto con extraordinaria convicción; no siendo susceptibles de ser sometidos a crítica racional, y su contenido se encuentra marcadamente alejado de la realidad y los patrones culturales de la persona que lo padece. Por lo mismo no son de tipo transitorio, sino que son persistentes, y experimentados como verdaderos, llevando al sujeto a actuar en relación con los contenidos de los mismos. En algunos casos los pacientes presentan un solo delirio de carácter insistente y preocupante. Suele aparecer en la mente del enfermo sin motivación determinada y el sujeto experimenta transformaciones y malestar con la realidad donde se encuentra. (Frith, C., Connie C., 1998)

La psiquiatría clásica menciona que la mayor parte de sujetos que desarrollan esquizofrenia manifiestan una conducta esquizoide, la misma que se caracteriza por una escasa sociabilidad, excentricidad, sensibilidad y excitación inapropiada. La persona experimenta una alteración en la relación con su ambiente, la misma que se pierde o se ve gravemente trastornada, la atención se vuelve dispersa o fija en la idea delirante, la conversación adquiere características de incoherencia y suele girar en torno a ideas obsesivas ajenas a la situación. Existen también síntomas de aislamiento social grave, irritabilidad cuando el sujeto es contradicho o interrumpido, comportamiento extravagante, intereses personales reducidos y escasos, conducta regresiva, e inactividad física; toda esta semiología suele ser agrupada con el nombre de comportamiento desorganizado. Se considera además que la imaginación y la percepción se funden y son proyectadas sobre el ambiente, los pacientes racionalizan las alucinaciones y los delirios, experimentan fuertes preocupaciones intrapsíquicas, apareciendo retraimiento, negatividad y estereotipias. Las alteraciones del pensamiento se encuentran marcadas por la presencia de pensamiento concreto, incapacidad de abstracción, sus observaciones se vuelven primitivas y concretas, su pensamiento guarda una gran exactitud literal, esto se debe a la fragmentación psíquica fundamental en la enfermedad (Wolff, 1950/2005).

Sluchevsky en Psiquiatria (1960 /1984), plantea que el síntoma cardinal de la esquizofrenia es el embotamiento de la sensibilidad, que se evidencia en un inicio con un cambio importante en la actitud del sujeto hacia sus seres más 
cercanos, hacia quienes la persona se muestra indiferente y distante, cuando no abiertamente agresivo. Este embotamiento paulatinamente va abarcando otros ámbitos de la vida del sujeto hacia casi todos sus intereses, llegando a alterar su actitud inclusive ante la alimentación, el cuidado personal y la sexualidad. Este embotamiento afectivo genera también reacciones de cólera o alegría incontroladas e inesperadas, provocando risas escandalosas e inmotivadas y ambivalencia afectiva. El sujeto va mostrando cada vez más indiferencia ante el medio natural y social y termina en un estado de aislamiento casi completo.

Asociado al embotamiento afectivo, encontramos los trastornos del pensamiento, caracterizados por razonamientos y reflexiones patológicas, centrados en intensos procesos de análisis de temas e intereses insignificantes, cargados de elementos filosóficos incomprensibles en el marco social de la persona y verborrea excesiva. El tercer síntoma fundamental será la abulia, que implica una reducción significativa de la actividad voluntaria del sujeto, llegando en estados avanzados y graves de la enfermedad a que el enfermo inclusive reduzca sus actividades fundamentales como la actividad alimenticia. El esquizofrénico, además, no pierde la orientación en el lugar, tiempo o personas que le rodean, ni ve afectada su memoria o inteligencia, que se conservan aunque fragmentadas y muchas veces sin una finalidad práctica específica. La atención se ve alterada, fundamentalmente la atención activa (Sluchevsky, 1960/1984).

El período de instauración de la esquizofrenia se caracteriza por una serie de síntomas que anticipan el desarrollo patológico pero que se encuentran en un estado de intensidad muy baja lo que dificulta un adecuado diagnóstico. Los rasgos fundamentales de este período son la presencia de ambivalencia afectiva, especialmente centrada en las personas cercanas al sujeto; extravagancia, es común la presencia de conductas extrañas, caprichosas, generalmente de carácter inútil y asociadas a ideas fijas; impenetrabilidad, que se manifiesta en una vida privada y psíquica sumamente hermética; el distanciamiento con las demás personas se encuentra determinado en esta etapa por la imposibilidad que las ideas ajenas influencien en la actividad y pensamientos de la persona; tenemos además el desapego, que se caracteriza por un paulatino alejamiento de las demás personas, perdiendo el medio social interés para el paciente, refugiándose en su impenetrable vida mental e ideas delirantes. Estas ideas delirantes se caracterizan por ser incorregibles, es decir que el paciente se encuentra convencido radicalmente de las mismas es imposible que las modifi- 
ReVista PUCE. ISSN 1390-7719. Núm.103. 3 de mayo de 2016 -

3 De nOViembre De 2016. ERnesto Flores SierRa. PP. 33-63

que en lo más mínimo; son irreversibles, puesto que incluso ante la imposibilidad material de las mismas, el paciente mantiene la intensidad de la idea delirante actuando acorde con ellas; presentan imposibilidad de contenido, puesto que generalmente reflejan una imposibilidad práctica inherente; finalmente presentan rasgos de invasividad, puesto que terminan abarcando la personalidad y el pensamiento de la persona en casi su totalidad, muchas veces convirtiéndose en los únicos ejes de toda su vida y actividad (Paladines, 2008).

Se ha descrito además que la esquizofrenia es una enfermedad con una altísima complejidad y variabilidad sintomática, es común que no todos los síntomas aparezcan al mismo tiempo, y que por lo general los síntomas más activos (síntomas positivos) solo se manifiesten durante las crisis psicóticas. Los síntomas negativos suelen pasar desapercibidos durante las primeras fases de la enfermedad, y generalmente son de baja intensidad, pero de larga duración en el tiempo, marcando la vida cotidiana de la persona esquizofrénica (Rodríguez Martínez, A., Castaño Ansis, J., 2011).

Los trastornos en la afectividad se caracterizan por un afecto restringido e inapropiado, consiste en un progresivo aplanamiento emocional, marcado por una disminución paulatina de la respuesta afectiva. En su aspecto inadecuado es común la presencia de afectos que no corresponden a las circunstancias. Puede aparecer también una pérdida de interés por aspectos que antes eran fundamentales para la vida de la persona, en especial por las relaciones sociales, lo que determinará la presencia de un comportamiento de aislamiento y autismo, así como al descuido personal y la falta de higiene. (Rodríguez Martínez, A., Castaño Ansis, J., 2011)

\section{AUTOPSIA PSICOLÓGICA DE CHRISTOPHER MCCANDLESS}

La autopsia psicológica es la reconstrucción de los rasgos sobresalientes de la vida de un sujeto, para comprender su personalidad, y qué papel jugaron estos rasgos y esta personalidad en las circunstancias de su muerte (Jiménez, 2001). Las circunstancias de la muerte de Christopher McCandless han quedado para el campo de la especulación forense. Quienes realizaron la autopsia determinaron que la causa de su fallecimiento fue inanición; sin embargo, su biógrafo Krakauer, planteó siempre la teoría de una intoxicación por el consu- 
mo de unas semillas de tubérculos con alto grado de toxicidad.

Krakauer, ha defendido siempre que el estado psicológico de McCandless no influyó en su muerte, la misma que fue, en opinión del autor un accidente; sin embargo, los conocimientos de psicología del periodista pueden llevarlo a opiniones fundadas en un análisis superficial de los datos vitales que él mismo recogió de las personas cercanas al sujeto de investigación y de sus diarios personales. La propuesta de la presente investigación consiste en demostrar la presencia en McCandless, de una personalidad esquizoide y una esquizofrenia prodrómica, mediante la realización de una autopsia psicológica a base de los datos que Krakauer nos provee en la biografía del autostopista.

La historia de la muerte de McCandles se inicia en abril de 1992, cuando este se interna solo en los bosques de Alaska.

En abril de 1992, Chris McCandless, de 24 años, se internó solo y apenas equipado por tierras de Alaska. Había regalado todo su dinero y abandonado su coche, y soñaba con una vida en estado salvaje. Cuatro meses más tarde, unos cazadores encontraron su cuerpo sin vida. (Krakauer, 2008)
La preferencia por la soledad, el aislamiento social, el abandono de las actividades sociales habituales, suele ser el primer indicador de la presencia de una fenomenología esquizoide, el deseo de alejarse voluntariamente del medio social, y aislarse totalmente de todo contacto con otros seres humanos, es un fenómeno que delata el inicial desarrollo de una enfermedad psicótica. El síntoma negativo de aislamiento social, constituye el indicador más crónico y permanente de la esquizofrenia. El deseo insistente de McCandless de alejarse de todo contacto social, es la primera evidencia que se encuentra para proponer la presencia de un cuadro esquizoide.

En el verano de 1990, tras graduarse en la Universidad Emory de Atlanta, McCandless desapareció. Cambió de nombre, donó a una organización humanitaria los 24.000 dólares que guardaba en su cuenta corriente, abandonó su coche y la mayor parte de sus pertenencias, y quemó todo el dinero que llevaba en los bolsillos. Luego, se inventó una nueva vida, pasó a engrosar las filas de los desheredados y marginados, y anduvo vagando por América del Norte en busca de experiencias nuevas y trascendentes. La familia no supo nada de su paradero o su suerte 
ReVista PUCE. ISSN 1390-7719. Núm.103. 3 de mayo de 2016 -

3 De noviembre de 2016. ERnesto Flores Sierra. PP. 33-63

hasta que sus restos aparecieron en Alaska. (Krakauer, 2008)

Se conoce que los síntomas psicóticos negativos, comienzan a desarrollarse con el inicio de la vida adulta del sujeto, los relatos de los familiares de McCandless comienzan a denotar rasgos de aislamiento social y conducta excéntrica entre los 18 y 24 años, precisamente la edad de inicio de la enfermedad, que llevan a que este desarrolle una conducta de aislamiento y vagabundeo, característica de las personas esquizofrénicas. El episodio de la "quema del dinero", si bien adquiere, un toque "romántico" dentro de una sociedad como la norteamericana, puede ser leído también como una conducta desorganizada, marcadamente alejada del campo de referencia cultural del sujeto, pues es además, por los relatos de familiares y amigos, una conducta asociada a la idea de una "malignidad esencial" del dinero, que nos revela la presencia de ideas particularmente excéntricas.

En cuanto a las relaciones con su familia, la niñez de McCandless y adolescencia no muestran datos particulares, y son relatadas como "tranquilas" por sus familiares, no parece haber dado ningún tipo de problemas importantes; sin embargo, estos rasgos que podrían considerarse como convencionales adquieren una importancia mayor cuando se aso- cian con una conducta de aislamiento y retraimiento social:

\begin{abstract}
«Chris era muy suyo, ya de pequeño —explica su hermana Carine, que nació tres años después-. No es que fuera antisocial, ya que siempre tuvo amigos y caía simpático a todo el mundo, pero podía salir al jardín y entretenerse solo durante horas. Parecía no necesitar los juguetes ni los amigos. Sabía estar solo sin sentirse solo.» (Krakauer, 2008)
\end{abstract}

La aclaración que realiza su hermana "no es que fuera un antisocial" revela que dicha conducta siempre fue observada por su familia, los rasgos de aislamiento social parecen haberse iniciado desde etapas tempranas de la vida del sujeto, estando acompañadas de un comportamiento tranquilo, nos hablan del proceso de formación de una personalidad típicamente esquizoide.

No se metía en problemas, siempre sacaba notas excelentes y hacía lo que se esperaba de él. Sus padres no tenían ningún motivo de queja, pero no lo dejaron tranquilo hasta que se salieron con la suya. No sé qué le dijeron, pero funcionó. Al final terminó yendo a Emory, pese a que seguía pen- 
sando que no tenía sentido, que era una pérdida de tiempo y dinero.» (Krakauer, 2008)

La semiología de la esquizofrenia, nos dice que en el pródromo de la enfermedad, se producen cambios bruscos afectivos concentrados en las personas más cercanas del sujeto, en el caso de McCandless tenemos varios relatos familiares que denotan la presencia de esta conducta:

Muchos aspectos de la personalidad de Chris confundían a sus padres. Podía ser generoso y cariñoso en extremo, pero también tenía un lado oscuro, caracterizado por la monomanía, la impaciencia y el ensimismamiento, rasgos que parecieron intensificarse durante el tiempo que estuvo en la universidad. (Krakauer, 2008)

La descripción de estos rasgos que "confundían a sus padres", que pueden ser descritos como asociados a un inicial embotamiento afectivo se corresponden con los síntomas que anuncian el desarrollo temprano de un cuadro psicótico. Rasgos que se acrecentarían durante el año que McCandless pasó en la universidad:

Durante el último año que pasó en Atlanta, Chris había vivido fue- ra del campus en una habitación monacal, amueblada con poco más que un delgado colchón extendido sobre el suelo, una mesa y unas cajas de cartón. Lo mantenía todo tan ordenado y sin mácula que parecía un barracón del ejército. Ni siquiera tenía teléfono, de modo que Walt y Billie no podían Ilamarlo (...) Durante su último año en Emory vivió fuera del campus, en una habitación espartana amueblada con cajas de cartón y un colchón en el suelo. Apenas veía a sus escasos amigos fuera de clase. Un catedrático le proporcionó una llave para que accediese de madrugada a la biblioteca, donde se pasaba la mayor parte del tiempo libre. (Krakauer, 2008)

El desapego del mundo real, que lleva al desarrollo e implantación de la esquizofrenia pueden observarse en estos relatos sobre la estancia del sujeto en la universidad, distanciamiento de los lazos sociales más cercanos; en este caso los lazos familiares, baja capacidad para sentir placer, conductas compulsivas no neuróticas, que además van de la mano con un distanciamiento, no solo de sus padres y familia, sino de todo tipo de contacto social, que llevan a la persona a la práctica de actividades solitarias: 
ReVista PUCE. ISSN 1390-7719. Núm.103. 3 de mayo de 2016 3 De noviembre de 2016. ERnesto Flores Sierra. PP. 33-63

Para su cada vez más reducido círculo de amigos, McCandless parecía volverse más impulsivo cada mes que pasaba. Tan pronto como terminaron las clases en la primavera de 1989, McCandless tomó el Datsun y emprendió otro de sus largos e improvisados viajes por carretera (Krakauer, 2008)

Esta conducta excéntrica es propia de un proceso de fragmentación que se experimenta en las primeras etapas de desarrollo del cuadro psicótico; primero tenemos la relatada impulsividad que llevaba al sujeto a distanciarse, cada vez con mayor frecuencia de todo tipo de contacto social, y además una tendencia permanente al abandono de todo tipo de conducta social dentro de su marco cultural. La ausencia de relaciones con miembros de su familia, como con sus compañeros de universidad, así como una conducta excéntrica con una fuerte tendencia a la soledad, nos hablan de la implantación de rasgos propios de las fases iniciales de la esquizofrenia.

Tras abandonar la universidad, cambiarse de nombre, perder su auto, McCandless se convierte en un autostopista que recorrió extensas regiones del oeste de Estados Unidos, realizando dicho recorrido solo, la mayoría del tiempo. De los relatos de las personas con quienes tomó contacto durante este período se pueden obtener datos importantes para la presente autopsia psicológica:

Su rostro poseía una rara elasticidad; podía mantenerse inmóvil e inexpresivo para transformarse al cabo de un instante en una enorme sonrisa bobalicona que desfiguraba sus facciones y ponía al descubierto unos dientes caballunos. Era miope y llevaba unas gafas con montura metálica. Parecía hambriento. (Krakauer, 2008)

La anterior descripción fue realizada por Wayne Westerberg, y recogida en el texto biográfico de Krakauer; en la misma se describe otra particularidad sintomática de la semiología negativa de la esquizofrenia, que tiene que ver con la presencia de risas inmotivadas, labilidad afectiva, y reacciones inapropiadas afectivas en relación con las circunstancias. La comunicación afectiva se ve afectada en la psicosis por el aparecimiento del embotamiento afectivo -descrito en la primera parte del estudio-, síntoma que afecta esta área específica de la socialización, y da a las sonrisas y demás reacciones emocionales del paciente esquizofrénico, su aspecto característico.

Era el trabajador más esforzado que haya visto jamás. Por penoso que fuera lo que le pidieras, él 
lo hacía. Los trabajos físicos más duros, desde limpiar el grano podrido hasta sacar ratas muertas del fondo de un agujero, tareas en las que terminas tan hecho una porquería que al final del día ni te reconoces. Nunca dejaba nada a medio hacer. Terminaba todo lo que empezaba. Se lo tomaba casi como un deber moral. Era lo que se dice alguien muy honrado, muy ético. Se exigía mucho a sí mismo. »Se notaba enseguida que era muy inteligente —reflexiona Westerberg mientras apura su tercera copa-. Leía mucho. Utilizaba palabras rebuscadas. En parte, creo que lo que pudo llevarlo a meterse en problemas era que pensaba demasiado. A veces se emperraba demasiado en querer entender el sentido del mundo, en desentrañar qué motivaciones podían tener las personas para ser tan malvadas las unas con las otras. En un par de ocasiones le comenté que era un error profundizar tanto en esos asuntos, pero Alex no paraba de dar vueltas y más vueltas a todo. Siempre tenía que saber cuál era la respuesta correcta a un problema antes de pasar al siguiente.» (Krakauer, 2008)
La descripción de las ocupaciones laborales de McCandless en el período que trabajó para Westerbeg, permiten apreciar la presencia de un cuadro patológico en proceso de instalación. La realización de tareas desagradables, que eran rechazadas por los demás trabajadores muestra una despreocupación marcada por olores, dolores, sensaciones, displacenteras, que nos orientan hacía la posible presencia del embotamiento afectivo descrito anteriormente. Además, la descripción anterior hace referencia a conductas que podrían ser enmarcadas dentro de los síntomas de ideación esquizoide; es decir, la realización de largas reflexiones "filosóficas" sobre temas intrascendentes, de carácter referencial, y que además tienen su correlato correspondiente en una expresión verbal cargada de rasgos verborreicos.

Muchas de las historias de "Álex" transcurrían en torno a un cinturón de cuero donde esculpió los momentos más importantes de su vida como autostopista, las mismas que eran repetidas una y otra vez con la carga de reflexiones insustanciales descritas en el párrafo anterior:

«Álex solía sentarse a la barra del Cabaret y pasarse horas leyéndonos lo que decía el cinturón - explica Westerberg-. Era como si estuviera traduciendo unos jero- 
ReVista PUCE. ISSN 1390-7719. Núm.103. 3 de mayo de 2016 3 De nOViembre De 2016. ERnesto Flores SierRa. PP. 33-63

glíficos. Cada uno de los dibujos que había grabado en el cuero tenía una larga historia tras de sí.» (Krakauer, 2008)

Uno de los eventos descritos por Westerberg, y recopilados en el libro de Krakauer, nos refiere indicios de conductas particulares que nos muestran signos de inadecuación a las normas de convivencia social, así como a la solución de problemas cotidianos que se podrían enmarcar dentro de la semiología descrita como incapacidad para el pensamiento abstracto y prevalencia del pensamiento concreto en la resolución de tareas:

McCandless tampoco estaba dotado de un excesivo sentido común. Muchos de quienes lo conocieron han comentado espontáneamente que parecía tener una gran dificultad para resolver problemas prácticos, como si los árboles le impidieran ver el bosque. "Alex no era un pasmado, entiéndame bien — prosigue Westerberg-. Pero en ocasiones parecía vivir en las nubes. Recuerdo que una vez fui a casa, entré en la cocina y noté un olor nauseabundo. El microondas apestaba. Lo abrí y el fondo estaba lleno de grasa rancia. Alex lo había utilizado para asar un pollo y no se le había ocurrido pensar que la grasa tenía que caer en algún sitio. No era que fuera demasiado perezoso para limpiarlo. Al contrario, Alex siempre lo tenía todo muy pulcro y ordenado. No se había dado cuenta de lo que pasaba, así de simple (Krakauer, 2008)

Si confiamos en la opinión de Westerberg, que parece estar confirmada por las demás informaciones provenientes de quienes lo conocieron, dichas conductas no se asocian a algún particular problema intelectual, sino a alteraciones en los procesos del pensamiento que impiden la resolución de problemas y es este otro importante patrón sintomático en los períodos prodrómicos de la esquizofrenia.

Observamos también en los relatos de Westerberg, el aparecimiento de ideas fijas que generan conductas excéntricas; la incapacidad de modificar una idea, frente a la argumentación racional, es también un rasgo de un pensamiento fragmentado:

«Cuando Álex había tomado una
decisión, no había modo de que
rectificara - dice Westerberg-.
Incluso le ofrecí comprarle un
billete de avión para Fairbanks, lo
que le habría permitido trabajar
otros diez días más y llegar a Alas- 
ka igualmente a finales de abril, pero repuso que no, que quería ir haciendo autostop, que volar sería como hacer trampas y le estropearía el viaje.» (Krakauer, 2008)

Otro elemento importante para la presente autopsia psicológica tiene que ver con el escaso interés que McCandless mostraba hacia las relaciones afectivas o la sexualidad; conductas propias de la esquizofrenia, y, que se desarrollan como consecuencia del aplanamiento afectivo, y la baja capacidad de sentir placer. Uno de los indicadores de inicio de un cuadro esquizoide es la ausencia de relaciones afectivas o relaciones sexuales, así como el abandono de todas o casi todas las actividades que el sujeto consideraba placenteras antes del aparecimiento de la enfermedad.

Así y todo, hay muy pocas pruebas de que fuera un adolescente sexualmente activo y, todavía menos, de que tras graduarse mantuviera relaciones sexuales con alguna mujer. (Por otro lado, tampoco hay pruebas de que alguna vez intimara sexualmente con un hombre.) Parece que McCandless se sentía atraído por las mujeres, pero que siempre o casi siempre permaneció célibe, tan casto como un monje. La castidad y la pureza moral fueron cualidades sobre las que McCandless reflexionó a menudo. (Krakauer, 2008)

En la época en la cual se encontraba vagando por las rutas de Estados Unidos tampoco presentó, hasta donde se puede indagar, acercamientos afectivos hacia otras personas, no estableció vínculos de pareja, y además desarrolló argumentaciones complejas sobre las razones de su aparente castidad:

«No recuerdo que Alex hablara de chicas nunca - dice Westerberg-, aunque un par de veces comentó que se casaría y tendría hijos. Daba la sensación de tomarse las relaciones sentimentales muy en serio. No era la clase de chico que empieza a salir con chicas solo para acostarse con ellas.» (Krakauer, 2008)

Inclusive Burres, una de las personas que proveyeron información sobre su vida en la carretera a Krakauer, menciona que ante el acercamiento de potenciales parejas jamás estableció un vínculo con estas personas, y nos refiere una tendencia a la soledad acompañada de una facilidad para establecer relaciones superficiales que pronto eran abandonadas. En las etapas iniciales de los cuadros psicóticos, el aislamiento social 
ReVista PUCE. ISSN 1390-7719. Núm.103. 3 de mayo de 2016 -

es progresivo, y se produce un paulatino alejamiento de la vida social, el mismo que muchas veces se caracteriza por el establecimiento de relaciones superficiales, en relación con períodos de soledad:

Álex se mostraba amable con ella, pero pensaba que era demasiado joven. Era incapaz de tomársela en serio. A ella le dolió en el alma que Álex se marchara.» Burres precisa que McCandless no era un solitario por más que rechazara los intentos de seducción de Tracy: «Se lo pasaba muy bien con la gente, disfrutaba. En el mercadillo hablaba como un desconocido con cualquiera que se le acercara. Debió de conocer a setenta $u$ ochenta personas, y siempre fue amable con todas y cada una de ellas. De vez en cuando necesitaba estar solo, pero no era un ermitaño. Al contrario, hacía mucha vida social, se relacionaba con todo el mundo. En ocasiones pienso que hacía acopio de compañía para los momentos en que sabía que no tendría a nadie.» (Krakauer, 2008)

En este período de su viaje, Burres relata nuevamente la presencia de la idea fija (posiblemente convirtiéndose en idea delirante) de viajar solo a Alaska, presentando dicha intención como una "odisea", descrita por la testigo como una "idea más fuerte que él"; esta característica muestra que la idea de alejarse definitivamente de la sociedad iba adquiriendo un carácter cada vez más mórbido.

$$
\begin{aligned}
& \text { «Cuando nos contó lo que iba } \\
& \text { a ser su "gran odisea en Alaska", } \\
& \text { como él la Ilamaba, pensé que } \\
& \text { había perdido el juicio —explica } \\
& \text { Burres-, pero estaba muy entu- } \\
& \text { siasmado. No hacía otra cosa que } \\
& \text { hablar del viaje; era más fuerte } \\
& \text { que él.» (Krakauer, 2008) }
\end{aligned}
$$

La gran mayoría del viaje de McCandless por las rutas de Estados Unidos lo hizo solo, y los datos del mismo han perdurado gracias al diario que llevaba, el mismo que es descrito por Krakauer en los siguientes términos:

\section{Por más que el diario esté escrito en tercera persona con un estilo afectado y artificioso y a menu- do tienda al melodrama, toda la información de que disponemos indica que no confundía realidad y ficción: para él, contar la ver- dad constituía un artículo de fe. (Krakauer, 2008)}

Como se mencionó en párrafos anteriores, la experiencia psicótica está 
marcada por un pensamiento alterado, el mismo que se manifiesta en el lenguaje; y en este caso en el lenguaje escrito; el diario de McCandless, y las posteriores anotaciones realizadas en el "autobús mágico", ya en Alaska, nos hablan de un lenguaje grandilocuente, artificioso, y que tiende a exagerar su experiencia cotidiana, orientando su discurso hacía su "odisea final".

Este viaje fue realizado en soledad, el factor de aislamiento social se evidencia en la mayor parte del recorrido de McCandless; este síntoma se corresponde con entradas cada vez más concisas y concretas que permiten leer un curso del pensamiento que va de lo artificioso y grandilocuente a lo concreto y específico; en especial en los períodos donde el aislamiento social se vuelve marcado. Se puede inferir, a base de estos indicios, que McCandless en los momentos en los cuales establecía vínculos sociales, por más momentáneos que estos fueran, desarrollaba un curso de pensamiento acelerado marcado por la grandilocuencia, mientras que en los momentos de aislamiento, este curso se reducía y se volvía concreto y fragmentado.

Tomó fotografías de tarántulas, de puestas de sol elegiacas, de dunas barridas por el viento, y del largo y solitario litoral. Las entradas del diario se hacen más con- cisas y superficiales. Durante el mes siguiente, apenas escribió un total de 100 palabras. El 14 de diciembre, cansado de tanto remar, desembarcó en una playa, dejó la canoa fuera del límite de la pleamar, trepó por un acantilado de arenisca y levantó su tienda de campaña en el extremo de una árida altiplanicie. Permaneció allí durante unos diez días, hasta que los fuertes vientos lo obligaron a buscar refugio en una cueva que se encontraba a medio camino de la escarpada pared del acantilado, donde se instaló otros diez días. Recibió el año nuevo contemplando la luna llena que se alzaba por encima del Gran Desierto, una inmensa extensión de 4.400 kilómetros cuadrados de dunas movedizas, el mayor desierto de arena de América del Norte (...) Durante 36 días no vio ni un alma, y subsistió con dos kilos de arroz y lo que conseguía sacar del mar, una experiencia que explica su posterior convencimiento de que podía sobrevivir en los bosques de Alaska con una ración diaria de comida igualmente exigua. (Krakauer, 2008)

El aislamiento social, el aplanamiento afectivo y la conducta desorga- 
ReVista PUCE. ISSN 1390-7719. Núm.103. 3 de mayo de 2016 -

nizada, generan en muchos casos de pacientes esquizofrénicos la conducta de "vagabundeo"; los pacientes escapan de sus hogares y viven en condiciones de marginalidad, hasta ser internados en hospitales psiquiátricos y reintegrados con sus familias. Según los datos de la biografía escrita por Krakauer, "Álex", pasaba gran cantidad de su tiempo conviviendo con vagabundos y marginales en zonas pauperizadas de las grandes urbes norteamericanas:

Aprendió a enterrar el dinero que llevaba consigo antes de entrar en una gran ciudad y desenterrarlo al salir. Así evitaba que lo atracase alguno de los desagradables personajes que rondaban por las calles y pasos elevados donde dormía. Según el diario, el 3 de febrero fue a Los Ángeles "para conseguir un documento de identidad y un trabajo»; pero «se siente muy incómodo viviendo en sociedad y experimenta la necesidad de volver de inmediato a la carretera». (Krakauer, 2008)

Se evidencia, que McCandless distribuía su tiempo entre el vagabundaje en las ciudades en zonas repletas de población marginal, y la soledad de las regiones no pobladas por donde se desplazaba. En algunos lugares urbanos, in- tentó trabajar; sin embargo, su conducta desorganizada impidió que se afirmara en alguno de estos lugares:

Lori Zarza, la ayudante del encargado, se formó una opinión un poco distinta de McCandless. «Con franqueza, me sorprendió que lo contrataran. Se limitaba a cumplir con su trabajo. Estaba en la cocina, en la parte de atrás, y era muy lento, incluso durante la hora punta del almuerzo. Daba igual que le metieras prisa. Tenías una cola de diez clientes en la caja y era incapaz de comprender por qué estabas encima de él. No lo captaba. Era como si estuviera perdido en su propio mundo.

»Sin embargo, era responsable. Nunca faltaba y siempre llegaba puntual, así que no se atrevían a despedirlo. Además, sólo le pagaban a 4,25 la hora. Con el montón de casinos que tenemos al otro lado del río que pagan de entrada a 6,25 la hora es difícil tener personal detrás del mostrador.

»No creo que saliera por ahí con algún compañero después del trabajo. Cuando hablaba, su tema de conversación eran los árboles, la naturaleza y cosas parecidas. Todos pensábamos que le faltaba un tornillo. (Krakauer, 2008) 
La conducta relatada por Zarza y descrita en el libro de Krakauer, revela ciertos rasgos de una conducta psicótica más acentuada; la vida en el desierto o en zonas de población marginal parecen haber acrecentado el déficit social, así como haber enlentecido a McCandless, mostrando rasgos sintomáticos que se han considerado históricamente a formas de esquizofrenia denominadas catatónicas, además de desarrollar conversaciones que a las demás personas les parecían extrañas y no haber mostrado rastros de socialización con quienes se encontraba trabajando. Los síntomas negativos parecen haberse instalado con fuerza en esta etapa, puesto que se nota en el texto que las personas que lo conocieron en esta etapa afirman ya que "le faltaba un tornillo"; mientras que las personas que lo habían conocido en otros momentos, aunque notaban su excentricidad aún lo consideraban como "normal". Para acentuar esta posibilidad, se encuentra en esta etapa referencias a un descuido personal notorio, malestar con las personas cercanas, e incapacidad para adecuarse a normas sociales o laborales mínimas:

Cuando empezó a trabajar, vivía en la calle y olía que apestaba. En McDonald's no está permitido entrar a trabajar sin un mínimo de higiene. Al final me encarga- ron que le dijera que se lavara más a menudo. Nuestras relaciones fueron de mal en peor desde el momento en que se lo dije. Y entonces los demás empleados empezaron a preguntarle si necesitaba algo, un poco de jabón, cosas así. Lo hacían sin mala intención, para ser amables, pero me di cuenta de que eso lo sacaba de quicio, aunque nunca lo demostraba. Al cabo de tres semanas, se marchó y no volvió.»

«Un buen chico, sí, muy buen chico - explica Charlie-. Aunque la gente no le gustaba demasiado. Tenía constantes cambios de humor. Sus intenciones eran buenas, pero creo que estaba cargado de complejos, ¿sabe lo que quiero decir? Le gustaba leer los libros de ese tipo que estuvo en Alaska, Jack London. No era muy hablador. Se ponía irritable, no quería que lo molestaran. Parecía uno de esos chavales que están buscando alguna cosa, algo, sin saber qué es. (Krakauer, 2008)

La esquizofrenia tiene un curso inestable, caracterizado por momentos en los cuales el sujeto experimenta incremento de la sintomatología, y otros en los cuales la misma disminuye y el estado funcional del paciente se recupera; 
ReVista PUCE. ISSN 1390-7719. Núm.103. 3 de mayo de 2016 -

sin embargo, mantiene las características esquizoides que marcan su conducta. El relato de la historia de McCandless nos habla de momentos en los cuales el incremento de la semiología negativa lo lleva hasta prácticamente la marginalidad y la apatía y momentos de un estado de adaptación significativo sin dejar de presentar rasgos psicóticos:

Sin embargo, McCandless no acampaba cerca de las fuentes, sino en el solitario entorno de la «bajada», a casi un kilómetro de distancia. Franz lo llevó hasta allí, charló un rato con él y luego regresó a la ciudad, donde vivía solo en un destartalado edificio de apartamentos que administraba a cambio de no pagar el alquiler. (Krakauer, 2008)

Podemos encontrar que McCandless habitaba en zonas alejadas de todo contacto social por períodos prolongados, inclusive cuando se instalaba en regiones con población cerca, elegía distanciarse voluntariamente de dicho contacto. La renuncia voluntaria al contacto social es característica del rasgo esquizoide de aislamiento social, diferente del rasgo fóbico social o evitativo, en el cual el sujeto se ve obligado por la ansiedad a distanciarse de otras personas o circunstancias sociales. En este caso, la renuncia al contacto social es voluntaria, y se encuentra asociada a un escaso o nulo interés en establecer relaciones con otras personas.

Pasan unos largos segundos
antes de que Franz se decida a
proseguir; cuando por fin vuelve
a hablar, levanta la vista al cielo
con los ojos entrecerrados. Du-
rante sus visitas, recuerda Franz,
no era raro que se pusiera de mal
humor y despotricase contra sus
padres, los políticos o la estupi-
dez endémica de la sociedad es-
tadounidense. Cuando era presa
de esos arrebatos, Franz apenas
decía palabra y lo dejaba perorar
por miedo a indisponerse con él.
(Krakauer, 2008)

Notamos que en este período de su viaje McCandless desarrolla ciertos rasgos de cambios violentos del estado de ánimo, relacionados con la semiología asociada al embotamiento afectivo. Hemos observado que dichas reacciones se presentaron primero contra sus padres, pero en este momento parecen haberse proyectado sobre la realidad misma. Los estudios sobre la psicosis, explicitados en la primera parte del artículo, describen esta proyección de contenidos negativos sobre la realidad y el distanciamiento de la persona esqui- 
zofrénica de la misma. El embotamiento afectivo y la alteración de pensamiento, se manifiestan en la expresión lingüística que como se menciona en el párrafo de la biografía del sujeto, donde se describen largos relatos agresivos de explicaciones del porqué del rechazo a la vida en su familia, a la sociedad y en general al marco de las relaciones sociales.

La idea fija de recluirse en los bosques de Alaska llevaría a que McCandless llegará a esta región a comienzos de abril del año 1992, para realizar su viaje hacia lo "salvaje" que planteaba había sido su "deseo" desde su infancia. Las advertencias de los locales sobre los riesgos de intentar adentrarse en las montañas en las fechas previstas por el autostopista, no modificaron su idea, lo cual revela que la misma se encontraba en un estado mórbido, es decir no podía modificarse por la crítica racional o por la evidencia empírica.

McCandless le confesó también que pensaba pasarse el verano solo en el monte, viviendo de lo que encontrara. «Dijo que era algo que deseaba hacer desde pequeño. Quería estar aislado, sin ver un avión, nada que le recordara la civilización, y demostrarse a sí mismo que podía arreglárselas solo, sin la ayuda de nadie.»
Stuckey y McCandless Ilegaron a Fairbanks el sábado 25 de abril. Primero efectuaron una breve parada en una tienda de comestibles. «Allí compró un saco de arroz. Luego me dijo que quería acercarse a la universidad para investigar las especies de plantas comestibles que encontraría en el bosque, ya sabe, bayas y cosas por el estilo. Le comenté que era demasiado pronto, que había más de medio metro de nieve y aún no crecía nada. Pero no me hizo caso. Estaba impaciente por salir de la ciudad.» (Krakauer, 2008)

Cuando McCandless estaba a punto de internarse en los bosques de Alaska, escribe la siguiente carta:

27 de abril de 1992

¡Recuerdos desde Fairbanks! Esto es lo último que sabrás de mí, Wayne.

Estoy aquí desde hace dos días. Viajar a dedo por el Territorio del Yukon ha sido difícil, pero al final he conseguido llegar. Por favor, devuelve mi correo a los remitentes. Puede pasar mucho tiempo antes de que regrese al sur. Si esta aventura termina mal y nunca vuelves a tener noticias mías, quiero que sepas que te consi- 
ReVista PUCE. ISSN 1390-7719. Núm.103. 3 de mayo de 2016 -

3 De noviembre de 2016. ERnesto Flores Sierra. PP. 33-63

dero un gran hombre. Ahora me dirijo hacia tierras salvajes. ÁLEX

El análisis de la misiva revela la idea del autor de no volver del lugar donde piensa recluirse del mundo, no habla de una "aventura" para regresar a la sociedad; nos habla de un distanciamiento total del medio social. El ser humano es un ser fundamentalmente social, la psicología patológica ha descrito desde hace siglos las consecuencias demenciantes del aislamiento, observado en niños abandonados, prisioneros, pacientes psiquiátricos, náufragos. El deseo de McCandless de abandonar voluntariamente la sociedad para no volver, como relata en la carta, nos habla de un rompimiento voluntario con la condición primaria de salud mental de todo ser humano, el contacto social.

La última persona que vio vivo a McCandless fue un habitante de la región, de apellido Gallien que lo llevó hasta la ruta de la estampida y que relata su encuentro de la siguiente manera:

Aun así, Gallien se inquietó. Álex reconoció que todo el alimento que llevaba en la mochila era un saco de arroz de cinco kilos. Su ropa y su equipo parecían exiguos en grado sumo para las duras condiciones de las tierras interiores, que en abril seguían sepultadas bajo una gruesa capa de nieve invernal. Las baratas botas de excursionista que el chico calzaba no eran impermeables ni termoaislantes. Su rifle era sólo del calibre 22; no podía confiar en un calibre tan pequeño si pensaba cazar grandes animales como el caribú o el alce, que era lo que tendría que comer si esperaba quedarse una larga temporada en aquellas montañas agrestes. No llevaba hacha ni raquetas, brújula ni repelente para insectos. La única ayuda de que disponía para orientarse consistía en un maltrecho mapa de las carreteras del estado, que había gorreado en una gasolinera. (Krakauer, 2008)

En este punto podemos notar que la idea de aislarse de la sociedad de McCandless estaba atravesada por una reflexión que puede definirse como anormal. Las condiciones de preparación era sumamente escasas; años antes había logrado sobrevivir en la costa oeste de los Estados Unidos comiendo arroz, pero evidentemente, las condiciones de los bosques fríos de Alaska demandan el consumo de alimentos ricos en grasa; una reflexión no psicótica, hubiese podido distinguir la evidente diferencia de necesidades calóricas, más aun conside- 
rando que McCandless se interna en los bosques cuando estos estaban cubiertos de nieve. Sus botas y vestido no eran tampoco los adecuados, de no ser por la generosidad de Gallien, podía haber muerto incluso antes por congelación, y el rifle que llevaba era más un arma recreativa que una herramienta de caza real. El mapa merece una atención especial, la idea de McCandless no era perderse sin mapa, de hecho, llevaba un mapa, pero un mapa obtenido en una gasolinera que era evidentemente inservible para internarse en el bosque. El curso y contenido del pensamiento se muestra evidentemente alterado. Es decir, el sujeto, percibía los fenómenos ambientales y sus necesidades (frío, hambre, necesidad de caza, necesidad de un mapa), pero dicha percepción estaba atravesada por una alteración del pensamiento que no le permitía enfrentarlos de una manera adecuada (poca comida y de baja carga calórica, rifle recreativo, ropa inadecuada, mapa inservible).

«No había manera de convencerlo de que no lo hiciera -recuerda Gallien-. Lo tenía todo muy claro. No atendía a razones. La única manera que se me ocurre de describirlo es que estaba ansioso. Se moría de ganas por llegar y emprender la marcha.» (Krakauer, 2008)
El relato de Gallien nos permite evidenciar además un factor característico de toda idea delirante, la incapacidad de ser sometida a crítica racional. Podríamos inclusive suponer que McCandless desconocía las particularidades de Alaska y había errado en la elección de su equipo, pero la confrontación racional del residente del lugar, en el caso de un pensamiento normal, hubiera hecho que el sujeto retrocediera ante la evidencia de la equivocación, y se equipara mejor para la aventura (común entre los cazadores de la zona). Sin embargo, la idea fija de internarse en el bosque para estar aislado de la sociedad, no puede ser sometida a crítica, y los intentos de Gallien no pueden disuadir a Alex de su intento, y termina internándose en la "senda de la estampida", con un equipo evidentemente deficiente.

McCandless decidió adentrarse en el monte con víveres insuficientes y un equipo que carecía de las herramientas que los habitantes de Alaska consideran imprescindibles para semejante excursión: un rifle de gran calibre, un mapa, una brújula y un hacha. Esto ha sido juzgado no sólo como una muestra de estupidez, sino, lo que es más grave todavía, como un pecado de arrogancia. (Krakauer, 2008) 
ReVista PUCE. ISSN 1390-7719. Núm.103. 3 de mayo de 2016 -

3 De nOViembre De 2016. ERnesto Flores SierRa. PP. 33-63

El duro juicio que sobre "Álex" mencionan los habitantes de Alaska nos remite a dos posibilidades, o bien asumimos que los errores fatales cometidos por McCandless tienen que ver con "estupidez" lo que no parece ser correspondiente con sus logros académicos previos, o tienen que ver con el aparecimiento incontenible de una conducta esquizoide que en conjunto con un pensamiento gravemente alterado, lo llevó a realizar una travesía con una preparación deficiente, en consonancia con la posible idea delirante de vivir una aventura salvaje en medio de la naturaleza. El diario de McCandless en esta época deja el estilo grandilocuente de los años anteriores y se convierte en un diario básicamente de los alimentos que encontró en el bosque subártico; este puede considerarse un indicador de una reducción del pensamiento abstracto y una implantación de un pensamiento concreto, característico del estado negativo de la alteración psicótica.

Las entradas del diario de McCandless contienen pocas reflexiones abstractas sobre la naturaleza; en realidad, apenas si encontramos en ellas reflexiones de ningún tipo. Las referencias al paisaje circundante son escasas.

Es más, tal como comenta Andrew Liske, el amigo de Roman, tras haber leído una fotocopia del diario, la mayor parte de las entradas tratan acerca de lo que comía.

- Apenas escribía sobre otra cosa que no fuese su alimentación.

Andrew no exagera. El diario es poco más que un recuento de las piezas de caza menor que logró abatir y las plantas comestibles que encontró. (Krakauer, 2008)

El último fragmento que denota un curso de pensamiento grandilocuente y acelerado, se puede encontrar en una inscripción hecha por McCandless en un autobús para cazadores que encontró en medio del bosque y que convirtió en su morada durante 114 días. La misma que reza lo siguiente:

HACE DOS AÑOS QUE CAMINA POR EL MUNDO. SIN TELÉFONO, SIN PISCINA, SIN MASCOTAS, SIN CIGARRILLOS. LA MÁXIMA LIBERTAD. UN EXTREMISTA. UN VIAJERO ESTETA CUYO HOGAR ES LA CARRETERA. ESCAPÓ DE ATLANTA. JAMÁS REGRESARÁ. LA CAUSA: "NO HAY NADA COMO EL OESTE.» Y AHORA, DESPUÉS DE DOS AÑOS DE VAGAR POR EL MUNDO, EMPRENDE SU ÚLTIMAY MAYOR AVENTURA. LA BATALLA 
DECISIVA PARA DESTRUIR SU FALSO YO INTERIOR Y CULMINAR VICTORIOSAMENTE SU REVOLUCIÓN ESPIRITUAL. DIEZ DÍAS Y DIEZ NOCHES SUBIENDO A TRENES DE CARGA Y HACIENDO AUTOSTOP LO HAN LLEVADO AL MAGNÍFICO E INDÓMITO NORTE. HUYE DEL VENENO DE LA CIVILIZACIÓN Y CAMINA SOLO A TRAVÉS DEL MONTE PARA PERDERSE EN UNA TIERRA SALVAJE.

ALEXANDER SUPERTRAMP MAYO DE 1992

Podemos notar la gran diferencia entre esta inscripción y las anotaciones de su diario que no pasan de referir cosas como "ardillas", "puercoespín" "semillas"; es decir, se nota el cambio de un curso acelerado de pensamiento a un estado de pensamiento lentificado y concreto. Se podría suponer, que el convivir con la naturaleza hubiese despertado en McCandless un espíritu literario de reflexiones abstractas, pero a base de la evidencia encontrada en sus diarios se deduce que el nivel de estrés que implica vivir a la intemperie, generó un acrecentamiento del cuadro negativo y fue enlenteciendo su psiquismo, hasta un estado abúlico que determinó su muerte por falta de alimentación.

El lugar donde McCandless se asentó es interesante para los fines de la presente autopsia psicológica, puesto que, como describe Krakauer, realmente no se encontraba alejado drásticamente de zonas pobladas o vías de paso de cazadores y turistas. Apenas se encontraba a 3 días de una carretera principal, en medio de un parque nacional y en una zona transitada por cazadores, tanto así, que estos habían conservado un viejo autobús minero, donde pernoctaban en las jornadas de caza, el mismo que sería el refugio de nuestro sujeto.

Aunque parezca irónico, los parajes solitarios que rodean el vehículo -el área cubierta de bosquecillos y maleza en que McCandless tomó la determinación de «perderse»— apenas pueden calificarse como tales según los parámetros que rigen en Alaska. La carretera de George Parks queda a menos de 50 kilómetros del lugar en dirección este. Sólo 25 kilómetros más al sur, detrás de una estribación de la cordillera Exterior, los coches de centenares de turistas entran en el parque del Denali por un camino que patruIlan los guardas forestales. $Y$ sin que el Viajero Esteta lo supiera, en un radio de unos diez kilómetros alrededor del autobús hay cuatro cabañas (aunque dio la casualidad de que en el verano de 1992 
ReVista PUCE. ISSN 1390-7719. Núm.103. 3 de mayo de 2016 -

ninguna de ellas estaba ocupada). Sin embargo, pese a la relativa proximidad entre el vehículo y la civilización, a efectos prácticos McCandless estaba aislado del resto del mundo (Krakauer, 2008)

El alejamiento de McCandless del medio social por lo tanto era fundamentalmente psíquico; a medida que pasaban los días, la escasa alimentación y las condiciones de vida fueron demacrando el cuerpo de "Álex", hasta que pasados dos meses de vida solitaria, decide salir de la zona donde se había refugiado y caminar hacia la carretera principal; al Ilegar al cruce del río Teklanika, se encuentra con este crecido, no puede cruzarlo y regresa al autobús donde moriría de inanición. Este acontecimiento se produce el 8 de julio, y McCandless muere aproximadamente el 20 de agosto, lo que significa que tuvo más de un mes para intentar salir, recorrer el río, cruzar el parque, o recorrer la ribera del río en busca de un cruce factible o poblaciones; sin embargo no lo hace, regresa al "bus mágico" y fallece por falta de alimentación. Para explicar esta inmovilidad, es necesario recordar nuevamente la semiología negativa de la esquizofrenia; los síntomas incrementan su acción sobre la conducta del sujeto cuando este se encuentra sometido a niveles altos de estrés. McCandless escribe en su diario que tras el fracaso al intentar cruzar el río, se siente "solo" y "asustado" lo que revela el incremento del estrés, y luego la inmovilidad que le termina costando la vida, vendría a ser consecuencia de este incremento y el probable déficit de actividad propio de los cuadros psicóticos, en este caso con el componente abúlico antes mencionado.

Si McCandless hubiera andado río arriba unos dos kilómetros, habría descubierto que el cauce se ensanchaba desgajándose en varios arroyos en un lugar donde las rocas formaban una especie de trenzado. Si hubiera examinado cuidadosamente aquel tramo del río, habría acabado por descubrir, quizás incluso por error, un punto en que el cauce sólo le llegaría a la altura del pecho. Sin duda, la fuerza de la corriente le habría hecho perder pie, pero nadando de un modo poco ortodoxo y brincando sobre el fondo es muy probable que hubiera conseguido cruzarlo antes de llegar a la garganta o sucumbir a la hipotermia(...) Puesto que carecía de mapa, el cable que colgaba sobre el río permaneció en un territorio ignoto. Así pues, en el momento de inspeccionar el violento caudal del Teklanika, McCandless llegó a la errónea 
conclusión de que es imposible alcanzar la orilla occidental. Pensó que la única vía de salida estaba cortada y regresó al autobús; una línea de acción razonable dado su desconocimiento del terreno. Ahora bien, si esto fue así, ¿por qué se quedó en el autobús hasta morir de hambre? ¿Por qué no intentó cruzar el Teklanika otra vez cuando llegó el mes de agosto, cuando el caudal se habría reducido de modo significativo y habría conseguido vadear el río con cierta seguridad? (Krakauer, 2008)

Se ha intentado explicar las causas del fallecimiento de McCandless por intoxicación, por envenenamiento, etc.; pero lo que es llamativo del caso, es la inmovilidad del sujeto. Síntoma que se corresponde con la abulia, la catatonia, el enlentecimiento del curso del pensamiento, y la incapacidad para el pensamiento abstracto. Como ejemplo, a tres horas de distancia del lugar donde fue hallado su cadáver, se encontraban tres cabañas de refugio para cazadores, equipadas con comida y adecuadas para refugio, a las cuales el sujeto nunca accedió por el estado abúlico en que parece haberse sumido por la condición de estrés

En resumidas cuentas, la salvación de McCandless parecía de- pender sólo de una caminata de tres horas río arriba. Esta triste ironía fue apuntada con reiteración en el período inmediatamente posterior al descubrimiento del cadáver(...)alguien entró en las tres cabañas y las saqueó. Los víveres terminaron por estropearse al quedar a merced de los animales y las inclemencias del tiempo (Krakauer, 2008)

La otra posibilidad radica en el hecho que McCandless posiblemente encontró las cabañas, teoría que es sostenida por Will Forsberg, quien propone que fue él mismo quien destrozó las cabañas que le hubieran podido servir de refugio y salvación. De ser cierta esta hipótesis se puede suponer la presencia, ya no solo de semiología negativa, sino de un posible brote psicótico que llevó a "Álex" a destruir una de las posibilidades de salvación, condenándose a morir de hambre

«Lo habían destrozado todo dice Will Forsberg-. Destruyeron cuanto no estaba clavado. Las lámparas aparecían rotas, así como los cristales de las ventanas, casi sin excepción. Arrastraron fuera camas y colchones y los amontonaron, arrancaron tablones del techo, agujerearon los bidones de gasóleo, movie- 
ReVista PUCE. ISSN 1390-7719. Núm.103. 3 de mayo de 2016 -

ron de sitio la estufa e incluso sacaron una gran alfombra para que se pudriera. La comida había desaparecido. Si Alex hubiese encontrado la cabaña, no le habría servido de gran cosa. O tal vez el problema es que la encontró.»

Forsberg considera que McCandless es el primer sospechoso del ataque que sufrieron las tres cabañas. (Krakauer, 2008)

Christopher McCandless fallecería a mediados de agosto en medio de los bosques, su cuerpo fue encontrado dos semanas después con claros indicios de una muerte por inanición, según su diario el intento de cruzar el río se produjo en el día 67, y falleció el día 114. Es decir, tuvo 47 días para salir de la región, buscar un poblado, alimentarse, encontrar fuentes calóricas; sin embargo decidió quedarse en el autobús hasta fallecer; la explicación que se propone es que el cuadro psicóti- co se incrementó por el estrés, eso le llevó a un estado de abulia, dejando por el mismo las actividades de caza y posiblemente las actividades alimenticias, muriendo finalmente de hambre.

El cuerpo de Chris McCandless se hallaba en un estado de descomposición tan extremo que fue imposible determinar con exactitud la fecha de su muerte, pero el médico forense no encontró señal alguna de heridas internas traumáticas ni fracturas óseas. En el cuerpo apenas quedaba grasa subcutánea y se apreciaba que los músculos se habían atrofiado durante los días o las semanas que precedieron a la muerte. En el momento de la autopsia, el cuerpo sólo pesaba 30 kilos. La forense dictaminó que la causa más probable del fallecimiento había sido el hambre. (Krakauer, 2008)

\section{CONCLUSIONES}

La sintomatología negativa de la esquizofrenia es un indicador fundamental del curso, desarrollo y cronicidad de la patología, a pesar que los síntomas floridos han sido más descritos y documentados, la semiología negativa es aquella que se presenta antes de los primeros brotes psicóticos, se mantiene durante los períodos de estabilidad y se manifiesta a lo largo de la vida del sujeto, permaneciendo en su fase residual.

Dichos síntomas suelen pasar desapercibidos por los familiares y personas cercanas de los sujetos, por cuanto 
en los momentos iniciales de la enfermedad no llegan a ser totalmente disruptivos, apenas la enfermedad se descubre cuando se produce el primer brote psicótico de síntomas positivos, en el cual se emite el diagnóstico; sin embargo, al momento de realizar la historia clínica generalmente se revela antecedentes de semiología negativa de larga duración en el tiempo.

El trastorno de personalidad esquizoide y la esquizofrenia tienen una altísima comorbilidad, y vamos a encontrar que por lo general el trastorno de personalidad se encuentra en la base de conducta del sujeto sobre la cual se desarrolla el trastorno psicótico. La división de carácter didáctico y semiológico que se realiza en el manual, y que sirve a propósitos diagnósticos, no implica que en la práctica clínica concreta dichos trastornos se encuentren separados, sino que suelen ser, en la mayoría de los casos, diferentes momentos de una misma alteración psíquica.

La autopsia psicológica es una herramienta fundamental para el trabajo del psicólogo en especial en el campo de la psicología clínica, forense o criminológica; la misma que se puede desarrollar con la información proveniente de fuentes primarias, secundarias, entrevistas, relatos, noticias, y que implica el desarrollo del método clínico hacia el campo de la investigación pericial.
Una posible explicación para el fatal desenlace de la aventura de Christopher McCandless es la presencia de un trastorno esquizofrénico con una carga de sintomatología negativa, la misma que se evidencia en los fuertes rasgos de personalidad esquizoide del sujeto, en el desarrollo de un aparente trastorno de personalidad en el inicio de la vida adulta, que lo llevó a un paulatino proceso de aislamiento social, para finalmente presentar una serie de brotes psicóticos, experimentados en diferentes momentos de su travesía, para finalmente ocasionar un estado de fragmentación psíquica incapacitante que lo estancó en los bosques de Alaska, lo cual ocasionó que muriera de inanición. Si bien los datos provienen de la biografía de Krakauer, y eso nos deja tan solo con una información fragmentaria; por lo mismo no se puede establecer un diagnóstico concluyente; sin embargo, podemos proponer la hipótesis de la presencia de rasgos esquizoides y síntomas esquizofrénicos que podrían explicar la muerte en el "bus mágico" de Alex Supertramp. 
ReVista PUCE. ISSN 1390-7719. Núm.103. 3 de mayo de 2016 -

\section{Bibliografía}

Andreasen, N. (1982). Negative v. positive schizophrenia: definition and validation. Arc.Gen. Psyquiatry (39), 789-794.

American Psiquiatric Association. (2014). DSM V.

Chinchilla Moreno, A., Quintero Gutiérrez del Álamo, J., Correas Lauffer, J. . (2011). Psicopatología y terapéutica psicofarmacológica de la esquizofrenia. Barcelona: Instituto de Altos Estudios Universitarios.

Frith, C., Connie C. (1998). Trastornos psicóticos: esquizofrenia, psicosis afectiva y paranoia. In A. y. Lazarus, Psicología anormal (pp. 63-103). México: Fondo de Cultura Económica.

Jimenez, I. (2001). La autopsia psicológica como instrumento de investigación. Revista Colombiana de Psiquiatría. Vol. XXX. N³, 271-276.

Krakauer. (2008). Hacia rutas salvajes. Barcelona: B, S.A.

Morrison, J. (2015). DSM-5: Guía para el diagnóstico clínico. México: Manual moderno.

Paladines, F. (2008). Curso básico de psicofisiología. Quito: Abya- Yala.

Rodríguez Martínez, A., Castaño Ansis, J. (2011). Conceptos generales sobre clínica, evolución y terapéutica de la esquizofrenia. Barcelona: Insituto de Altos Estudios Universitarios.

Sluchevsky, L. (1960/1984). Psiquiatría . México: Grijalbo.
Wolff, W. (1950/2005). Introducción a la psicopatología. México: Fondo de Cultura Económica 
